\title{
Original
}

\section{Circulating levels of miR-146a and IL-17 are significantly correlated with the clinical activity of Graves' ophthalmopathy}

\author{
Hongfa Wei*, Meiping Guan*, Yan Qin, Cuihua Xie, Xiajun Fu, Fang Gao and Yaoming Xue \\ Department of Endocrinology \& Metabolism, Nanfang Hospital, Southern Medical University, Guangzhou 510515, China
}

\begin{abstract}
Graves' ophthalmopathy (GO) is a common autoimmune disease that is difficult to deal with due to limited clinical evaluation methods. Recently miR-146a and Interleukin-17 (IL-17) have been found to be involved in autoimmune disorders and correlated with disease activity. However, it is unclear whether they are involved in Graves' ophthalmopathy (GO). The aim of this study is to investigate the correlation of circulating levels of miR-146a and IL-17 with clinical activity in GO patients. Fifty-seven study subjects were enrolled in four groups according to the corresponding criteria: active-GO, inactive-GO, Graves disease (GD) without ophthalmopathy, and healthy control group. The circulating levels of miR-146a and IL-17 were determined by qRT-PCR and ELISA, respectively. Serum IL-17 levels of GD, inactive-GO, and active-GO groups were all significantly higher than that of control (all $P<0.001$ ). Active-GO group had significantly higher IL-17 level than inactive-GO and GD groups ( $P=0.024$ and $P=0.001$, respectively). Active-GO and inactive-GO group had significantly lower miR-146a expressions than control $(P<0.05)$. Active-GO group had significantly lower miR146a than inactive-GO group $(P<0.05)$. Serum levels of IL-17 and miR-146a were both significantly correlated with clinical activity score (CAS) in GO patients $(P<0.001, P<0.001$, respectively). There was a significant negative correlation of circulating miR-146a expression with serum IL-17 levels $(P<0.01)$. These findings indicated that circulating levels of miR-146a and IL-17 may be potential biomarkers of active GO, and may play a key role in the progression of GO.
\end{abstract}

Key words: Graves' ophthalmopathy, microRNA, Interleukin-17, Clinical activity score

GRAVES' OPHTHALMOPATHY (GO) is a relatively common eye disease whose exact etiology and underlying mechanism still remain to be clarified. It is also called thyroid-associated ophthalmopathy (TAO), indicating that it is an organ-specific autoimmune disease [1]. Increasing evidence has accumulated to show that microRNAs (miRNAs), a class of short non-coding RNAs, play an important negative regulatory role in the process of autoimmune disease [2]. MiRNAs suppress gene expression by binding to the 3'-untranslated region (UTR) of target miRNAs, which presents an entirely new approach to posttranscriptional regulation of gene expression. In 2008, Mitchell et al. found that miRNAs were present in human plasma in a

Submitted May 24, 2014; Accepted Jul. 18, 2014 as EJ14-0246

Released online in J-STAGE as advance publication Aug. 7, 2014

Correspondence to: Yaoming Xue, Department of Endocrinology \& Metabolism, Nanfang Hospital, Southern Medical University, Guangzhou 510515, China. E-mail: xueyaoming999@126.com *Contributed equally.

The authors stated that there are no conflicts of interest regarding the publication of this article and have nothing to declare. remarkably stable form that was protected from endogenous RNase activity [3], indicating that they are likely involved in the immune response, such as inflammation and autoimmune disease.

Since then MiR-146a has been found to participate in the pathogenesis of autoimmune diseases such as rheumatoid arthritis (RA), systemic lupus erythematosus (SLE), and multiple sclerosis (MS) $[4,5,6]$. In innate immune reaction, miR-146a plays a key role in the regulation of inflammation through down-regulation of target gene (IRAK1) [7, 8, 9]. However, it is unclear whether miR-146a plays a role in the activity of GO.

Recently, miR-146a and IL-17 have been attracting more and more attention in autoimmune diseases.

Abbreviations: CAS, Clinical activity score; FT3, Free triiodothyronine; FT4, Free thyroxine; GD, Graves' disease; GO, Graves' ophthalmopathy; IL-17, Interleukin-17; IRAK1, Interleukin 1 receptor kinase; RA, Rheumatoid arthritis; SLE, Systemic lupus erythematosus; TAO, Thyroid-associated ophthalmopathy; TRAb, Thyroid hormone receptor antibody; TSH, Thyroid stimulating hormone. 
IL-17 has been found to be correlated with miR-146a in rheumatoid arthritis (RA) patients [10] and the serum IL-17 level in GO patients was reported to be associated with the activity of GO [11]. However, these studies did not include data on Grave's disease (GD) without ophthalmopathy. Thus, it is unclear whether serum IL-17 was a specific biomarker of active GO, or whether there was any correlation between miR-146a and IL-17 in GO patients.

The expression pattern of miR-146a in the plasma of $\mathrm{GO}$ patients thus remains unclear. In the present study, we enrolled fifty-seven subjects, including GD, inactive-GO, active-GO patients and healthy controls, to determine the circulating levels of miR-146a and IL-17 and to analyze their correlations with clinical activity score (CAS).

\section{Materials and Methods}

\section{Study subjects}

This study was approved by the ethics committee of Nanfang Hospital, Southern Medical University. Fiftyseven subjects were enrolled from Nanfang Hospital, Southern Medical University during the period from February 2012 to November 2013. Informed consent was obtained by the patients and control subjects.

Subjects were divided into four groups: GD group, active-GO group, inactive-GO group, and healthy control group according to the following criteria. (1) GD without ophthalmopathy group (GD group, $\mathrm{n}=15,8$

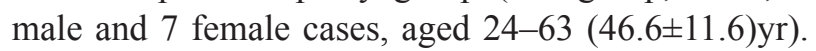
These patients were diagnosed as GD by typical symptoms of hyperthyroidism and laboratory examination of free triiodothyronine (FT3), free thyroxin (FT4), thyroid stimulating hormone (TSH), and thyroid hormone receptor antibody (TRAb), ruling out other kinds of hyperthyroidism such as autonomous hyperfunctioning adenoma. (2) Active-GO group ( $\mathrm{n}=14,5$ male and 9 female cases, aged 25-64 (40.3 \pm 12.6$) y r)$, with CAS of 3 points or more. Diagnostic criteria included primary disease, ocular symptoms and signs, laboratory examination (FT3, FT4, TSH, TRAb) and radiographic findings, excluding other causes of exophthalmos, other immune system disorders, other metabolic and infectious diseases, and patients with malignant tumors. (3) Inactive-GO group $(\mathrm{n}=13,6$ male and 7 female cases, aged 27-63 (38.5 \pm 11.3$) y r)$. The diagnosis and exclusion standard were the same as with active-GO group, but the CAS score was less than 3 points. None of the patients in these groups were treated by corticosteroids any other immunosuppressive therapy. (4) Healthy control group $(\mathrm{n}=15,8$ male and 7 female cases, aged 16-71 (44.3 \pm 16.2$) y r)$, without thyroid disease, autoimmune disease, liver disease or history of immunosuppressive therapy.

\section{Sample preparation}

Blood samples were obtained in the morning following $12 \mathrm{hr}$ of fasting. They were collected into empty tubes and immediately stored on ice at $4{ }^{\circ} \mathrm{C}$. The plasma was then separated by centrifugation at $1,500 \mathrm{rpm}$ for $10 \mathrm{~min}$. The serum was separated from the cells by centrifugation at 3,000 rpm for $10 \mathrm{~min}$. Plasma and serum samples for the measurement of miR-146a and IL-17 levels were stored at $-80^{\circ} \mathrm{C}$ until use.

\section{Measurement of serum IL-17 levels}

Serum levels of IL-17 were determined by human cytokine ELISA kits (Shanghai Joyee Biotechnics Co., Ltd., Shanghai, China) according to manufacturer's instructions. The detection limit of the IL-17 assay was $1800 \mathrm{pg} / \mathrm{L}$ and the intra-assay and inter-assay coefficients of variation were less than $10 \%$ and $15 \%$, respectively.

\section{Determination of circulating miR-146a expression}

RNA was isolated from plasma samples as previously described [3]. Total RNA was isolated from freshly obtained plasma using the Trizol kit (Takara Biotechnology (Dalian) Co., Ltd, Japan), in accordance with the manufacturer's protocol. Briefly, to extract the total RNA, $1000 \mu \mathrm{L}$ plasma was spun at $12,000 \mathrm{rpm}$ for $10 \mathrm{~min}, 4^{\circ} \mathrm{C}$. $1 \mathrm{~mL}$ Trizol (Takara Biotechnology (Dalian) Co., Ltd, Japan) was added into the tube which contained the plasma at room temperature. Then 0.2 $\mathrm{mL}$ chloroform was added into the same tube, shake it for $15 \mathrm{sec}$. The tube was spun at $12,000 \mathrm{rpm}$ for $10 \mathrm{~min}$, $4{ }^{\circ} \mathrm{C}$. The supernatant in the tube was carefully removed to the new tube. The isopyknic isopropyl alcohol was then added into the new tube above and was spun at $12,000 \mathrm{rpm}$ for $10 \mathrm{~min}, 4^{\circ} \mathrm{C}$. The supernatant in the tube was removed. Add $1 \mathrm{~mL} 75 \%$ DEPC alcohol into the tube to wash precipitation. And the tube was spun again at $12,000 \mathrm{rpm}$ for $5 \mathrm{~min}, 4^{\circ} \mathrm{C}$. The liquid of the tube was removed. Add $30 \mu \mathrm{L}$ RNase free water into the tube to dissolve RNA. Store the RNA at $-80^{\circ} \mathrm{C}$.

Reverse transcription kit (Takara Biotechnology (Dalian) Co., Ltd, Japan) was used to generate cDNA from the total RNA. The reactive conditions were 
$37^{\circ} \mathrm{C}$ for $15 \mathrm{~min}, 85^{\circ} \mathrm{C}$ for $5 \mathrm{sec}, 4^{\circ} \mathrm{C}$ for $5 \mathrm{sec}$. Then the products were collected for real time quantitative PCR (qRT-PCR). TAKARA SYBR ® Premix Ex Taq kit which contained $12.5 \mu \mathrm{L}$ SYBR ${ }^{\circledR}$ Premix Ex Taq TM, 0.2 $\mu$ L PCR Forward Primer, 0.2 $\mu$ L PCR Reverse Primer, $3.0 \mu \mathrm{L}$ cDNA and $9.1 \mu \mathrm{L} \mathrm{dH} 2 \mathrm{O}$ was used for qRT-PCR. The conditions of the reaction were $94^{\circ} \mathrm{C}$ for $2 \mathrm{~min}, 94^{\circ} \mathrm{C}$ for $30 \mathrm{sec}, 55^{\circ} \mathrm{C}$ for $30 \mathrm{sec}, 72^{\circ} \mathrm{C}$ for $30 \mathrm{sec}$. The primers of miR-146a used were: Forward: CAC TCC AGC TGG GTG AGA ACC TCA ACT GGT GTC GTG GA. Reverse: CTC AAC TGG TGT CGT GGA GTC GGC AAT TCA GTT GAG AAC CCA TG. U6 as Control: Forward: CTC GCT TCG GCA GCA CA; Reverse: AAC GCT TCA CGA ATT TGC GT. Relative quantification of miR-146a expression was calculated with the $2-\Delta \Delta \mathrm{Ct}$ method. In this way, the values of miR-146a expression obtained by qRT-PCR could be normalized using U6.

\section{Other parameters}

Serum FT4, FT3 and TSH concentrations were measured by electrochemiluminescence (commercial kits by Roche, Switzerland; normal values in our laboratory: $0.89-1.76 \mathrm{ng} / \mathrm{dL}$ and $2.5-4.2 \mathrm{pg} / \mathrm{mL}$ for FT4 and FT3, respectively; $0.55-4.78 \mathrm{mIU} / \mathrm{L}$ for TSH). TRAb was measured by the corresponding immunoradiometric assay kit by RSR Ltd. Co., Cardiff, U.K.; normal values were less than $10 \%$. Intra- and interassay coefficients of variation, for all assays, were less than $10 \%$ and less than $20 \%$, respectively. They were all measured by the laboratory of Nanfang Hospital.

\section{Statistical analysis}

Data were expressed as mean \pm SD using SPSS 13.0 for statistical analysis. Statistical significance was determined by one-way ANOVA. Differences of measurement data among the groups were analyzed by testing homogeneity of variances. LSD (least significant difference) test was used under an equal condition. While the homogeneity of variances was not equal, Dunnett's T3 test was used to compare the differences between groups and Spearman rank correlations were calculated between the nonparametric data.

\section{Results}

Fourteen active-GO patients, 13 inactive-GO patients, 15 GD patients, and 15 healthy controls were enrolled in this study. There were no significant differences in age, sex, degrees of exophthalmus, or levels of FT3, FT4, TSH or TRAb $(P>0.05)$ as shown in Table 1. The active-GO group had higher CAS than the inactive-GO group $(P<0.001)$.

Serum IL-17 levels of GD group $(\mathrm{n}=15)$, inactiveGO $(n=13)$ and active-GO group $(n=14)$ were all significantly higher than that of control group $(\mathrm{n}=15)$ (all $P<0.001$ ). The active-GO group had a significantly higher IL-17 level than the inactive-GO and GD group ( $P=0.024, P=0.001$ respectively). However, there was no significant difference between inactive-GO group and GD group $(P=0.104)$, as shown in Table 2. Serum IL-17 level was significantly correlated with CAS in GO patients ( $\mathrm{n}=27, \mathrm{r}=0.868, P<0.001)$ (Fig. 1 ).

There was no significant difference in circulating miR-146a expression between GD group and control group $(P=0.197)$. But the active-GO group and the inactive-GO group had significantly lower miR-146a expressions than the control group ( $P=0.002, P=0.009$ respectively). The active-GO group had significantly lower levels of miR-146a than the inactive-GO group $(P=0.039)$ (Fig. 2A). There was a significantly negative correlation between circulating miR-146a and CAS $(\mathrm{n}=27, \mathrm{r}=-0.738, P<0.001)$ (Fig. 2B). There was also a significant negative correlation between circulat-

Table 1 Comparison of clinical characteristics of each group

\begin{tabular}{lcccccc}
\hline Group & $\begin{array}{c}\text { Control } \\
(\mathrm{n}=15)\end{array}$ & $\begin{array}{c}\mathrm{GD} \\
(\mathrm{n}=15)\end{array}$ & $\begin{array}{c}\text { Inactive-GO } \\
(\mathrm{n}=13)\end{array}$ & $\begin{array}{c}\text { Active-GO } \\
(\mathrm{n}=14)\end{array}$ & F/t-value & $P$-value \\
\hline Sex (male/female) & $8 / 7$ & $8 / 7$ & $6 / 7$ & $5 / 9$ & 0.379 & 0.768 \\
Age (years) & $44.3 \pm 16.2$ & $46.6 \pm 11.6$ & $38.5 \pm 11.3$ & $40.3 \pm 12.6$ & 1.139 & 0.342 \\
Free T3 $(\mathrm{pg} / \mathrm{mL})$ & $\mathrm{NA}$ & $7.83 \pm 5.62$ & $5.44 \pm 2.52$ & $7.47 \pm 5.02$ & 0.964 & 0.391 \\
Free T4 $(\mathrm{ng} / \mathrm{dL})$ & NA & $5.15 \pm 3.09$ & $3.13 \pm 3.26$ & $4.09 \pm 4.33$ & 1.063 & 0.356 \\
TSH $(\mathrm{mIU} / \mathrm{L})$ & NA & $0.0082 \pm 0.01459$ & $0.0898 \pm 0.1945$ & $0.0372 \pm 0.0576$ & 1.813 & 0.177 \\
TRAb $(\%)$ & NA & $80.67 \pm 20.47$ & $70.49 \pm 22.95$ & $74.25 \pm 20.08$ & 0.674 & 0.517 \\
Exophthalmos $(\mathrm{mm})$ & NA & NA & $19.83 \pm 2.44$ & $19.61 \pm 2.03$ & 0.243 & 0.810 \\
CAS & NA & NA & $1.69 \pm 0.480$ & $4.21 \pm 0.975$ & 8.618 & $<0.001$ \\
\hline
\end{tabular}

Values are mean \pm SD. NA, not applicable. 
Table 2 Serum levels of IL-17 in each group

\begin{tabular}{ll}
\hline Group & $\mathrm{IL}-17(\mathrm{pg} / \mathrm{L})$ \\
\hline Controls $(\mathrm{n}=15)$ & $1859.3 \pm 438.3^{\# \#}$ \\
$\mathrm{GD}(\mathrm{n}=15)$ & $5266.9 \pm 1781.6^{* * * \#}$ \\
Inactive-GO $(\mathrm{n}=13)$ & $6698.9 \pm 1214.7^{\text {***\#}}$ \\
\hline Active-GO $(\mathrm{n}=14)$ & $9388.1 \pm 2811.1^{* * *}$ \\
\hline F value & 96.63 \\
$P$ value & $<0.001$ \\
\hline
\end{tabular}

Values are mean $\pm \mathrm{SD} .{ }^{* * *} P<0.001$ vs. Controls, ${ }^{\#} P<0.05$ vs. Active-GO, ${ }^{\#} P<0.01$ vs. Active-GO.

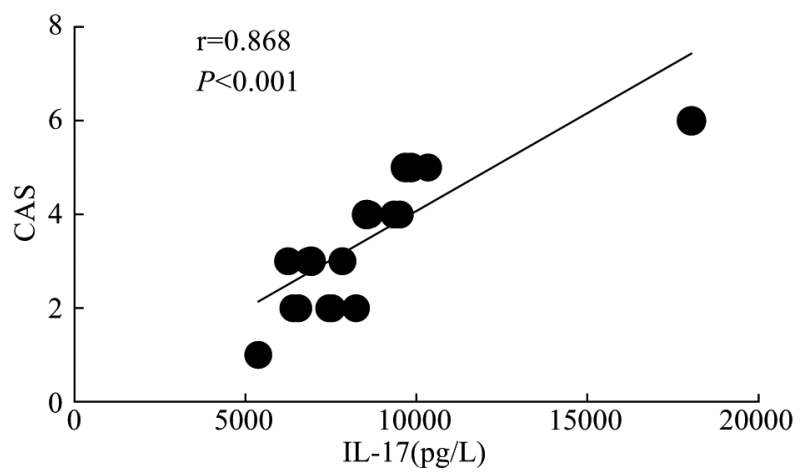

Fig. 1 Correlation between CAS and IL-17 levels in GO patients. Levels of IL-17 in serum positively correlated with CAS ( r $=0.868, P<0.001)$ in GO patients.
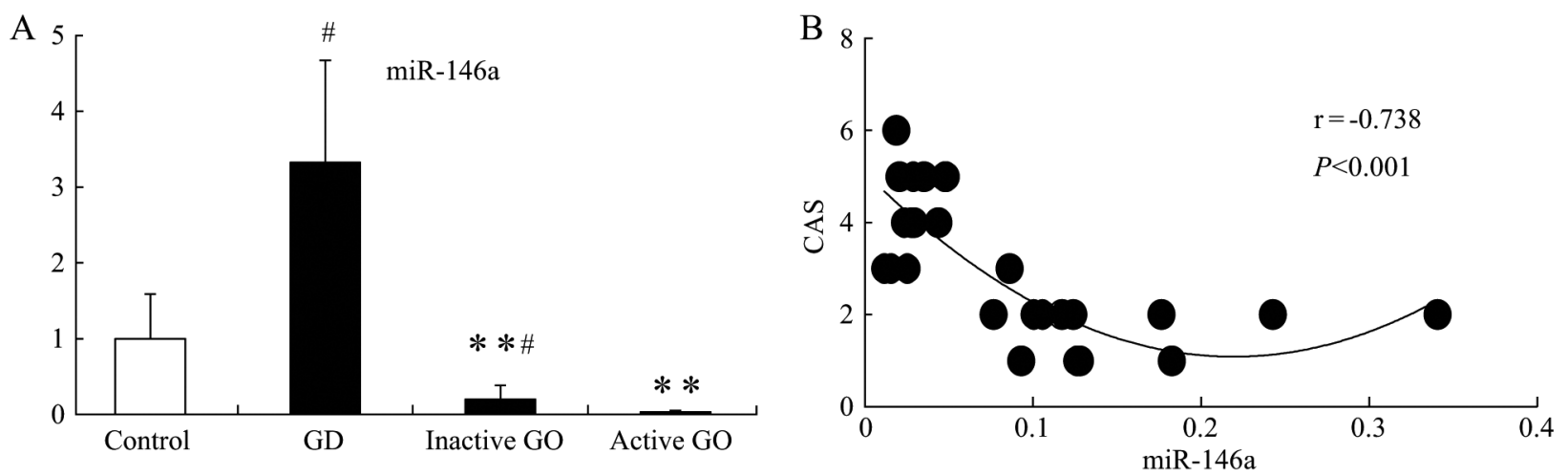

Fig. 2 Relative expression of miR-146a in plasma and the association with CAS. ${ }^{* *} P<0.01 v s$. Control group, ${ }^{\#} P<0.05$ vs. Active GO group. Relative expression of miR-146a (Fig. 2A) in plasma of GD group had no statistical difference $(P=0.179)$ with normal control group. Active GO group and inactive GO group were significantly lower than control group, the differences were statistically significant $\left({ }^{* *} P=0.002,{ }^{* *} P=0.009\right.$, respectively). Expression of miR-146a of active GO group was lower than inactive GO group expression ( ${ }^{\#} P=0.039$ ). Relative expression of miR-146a (Fig. 2B) in plasma of GO patients negatively correlated with CAS (r=-0.738, $P<0.001)$.

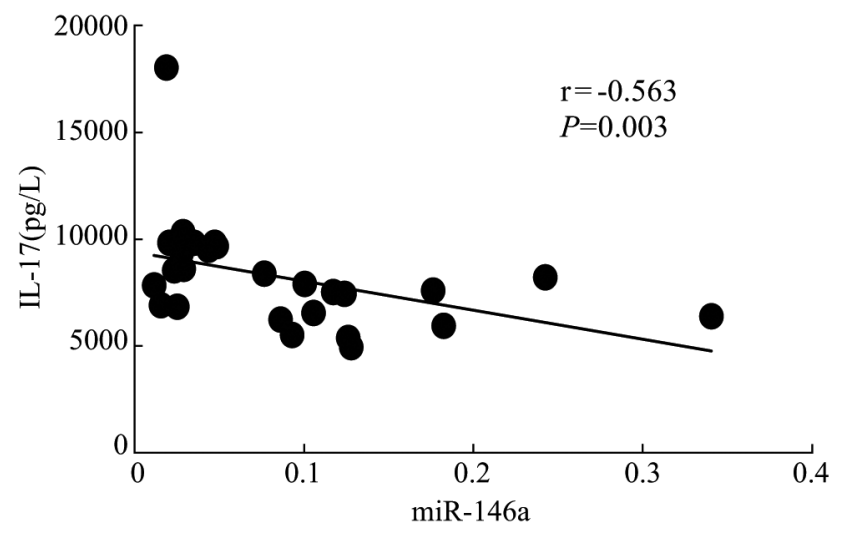

Fig. 3 Correlation between expression of miR-146a and IL-17 levels in GO patients. In GO patients, the relative expression of miR-146a in plasma was negatively correlated with IL-17 in serum ( $\mathrm{r}=-0.563, P=0.003)$. ing miR-146a expression and serum IL-17 level ( $\mathrm{n}=27$, $\mathrm{r}=-0.563, P=0.003)$, as shown in Fig. 3.

\section{Discussion}

GO, especially active GO, has long been a great challenge to endocrinologists. Active GO has been found to give priority to Th1 cells immune response, while inactive GO mainly gives priority to Th2 cells immune response [12]. Most recently a set of CD4 + effect $\mathrm{T}$ cells subgroup, namely, the Th17 cells, which are completely different from Th1 and Th2 cells in terms of biological functions [13] and characterized by their ability to produce large numbers of IL-17, has been found to be closely correlated with autoimmune disorders [14]. IL-17 acts as a proinflammatory cyto- 
kine to induce target cells to produce chemotactic factors and increase colony stimulating factors.

Accumulated evidence has shown that there are high serum IL-17 levels in a variety of autoimmune diseases, leading researchers to conjecture that IL-17 may be involved in the pathogenesis of various autoimmune disorders [15]. Serum IL-17 level is significantly higher in patients with SLE than healthy people and is positively correlated with the clinical activity of SLE [16]. IL-17 levels in serum and joint fluid of patients with RA were associated with the severity of the disease [17]. Patients with MS and asthma were also found to have significantly higher serum IL-17 levels than normal $[18,19]$. Our study showed that the serum IL-17 level in active-GO patients significantly increased compared with that of inactive-GO group, and was positively correlated with CAS in GO patients. These results are consistent with prior studies [11], which also demonstrated that IL-17 is a specific indicator of active GO. However, patients with GD without ophthalmopathy were not involved in their study. In our study we found that there was no significant difference between GD and inactive-GO group, which implied that high IL-17 level may be a specific biomarker of active-GO, indicating that IL-17 may participate in the etiology and development of GO.

The role of microRNAs in autoimmune diseases has been attracting increasing attention among researchers. Most recently miR-146a has been found to negatively regulate cytokines [20]. By targeting tumor necrosis factor receptor related factor 6 (TRAF-6) and interleukin 1 receptor kinase (IRAK1), miR-146a can negatively control LPS-TLR4 signaling pathways dependent on NF-kappa B [9]. IRAK1 was considered to play a key role in regulating the differentiation process of Th17 cells [21]. The miR-146a expression in peripheral blood mononuclear cells (PBMCS) of RA patients was obviously higher than that of healthy controls [22]. In addition, the miR-146a in RA synovial tissues was also significantly elevated [23]. Circulating miR-146a levels markedly declined in SLE compared with healthy controls [24]. MiR-146a was significantly decreased in the thyroid tissue of GD [25]. By contrast our results indicated that there was no significant difference in circulating miR-146a expression between GD group and control group in plasma, and that the circulating miR-146a expression in GO patients was not only significantly reduced but also negatively correlated with the clinical activity of GO. The discrepancy in the expression patterns of miR-146a among autoimmune diseases may be attributed to the complex mechanisms of the different autoimmune diseases.

There is a close correlation between miR-146a, IRAK1, and IL-17. The expressions of miR-146a and IL-17 in vitiligo patches and peripheral mononuclear cells of psoriatic patients were found to be significantly increased compared to those of the control group, whereas the target gene IRAK1 expression was expressed differentially in lesions and peripheral blood [26]. The inability of miR-146a to inhibit IRAK1 may contribute to the inflammatory process of psoriasis. In patients with RA, there was also a correlation between miR-146a and IL-17, with the IRAK1 in T cells which produce IL-17 being negatively regulated by miR-146a [10]. In our study, we found that the miR-146a of GO patients was decreased and negatively correlated with IL-17 levels. We hypothesized that the weakened ability of miR-146a, which further caused the increase of IL-17, may have participated in the progression of GO.

In conclusion, our findings indicated that circulating miR-146a expression and IL-17 level may be potential biomarkers of active GO. Further studies are warranted to explore how miR-146a interacts with IL-17 in the progression of GO.

\section{Competing Interests / Financial Disclosure}

Nothing to declare.

\section{Acknowledgements}

We wish to thank the Thyroid Study staff members (Lingling Xu, Linna Li, Ling Wang) at the Department of Endocrinology \& Metabolism, Nanfang Hospital, Southern Medical University for their involvement in the study.

\section{Authors' Contributions}

Yaoming Xue, Hongfa Wei and Meiping Guan designed the study. Hongfa Wei measured the serum samples. Cuihua Xie and Xiajun Fu assessed the clinical activity score (CAS) of GO patients. Yaoming Xue, Chenzhong $\mathrm{Li}$ and Fang Gao enrolled the subjects. Hongfa Wei and Meiping Guan analyzed the data and wrote the manuscript. Meiping Guan and Yaoming Xue supervised the study and revised the manuscript. 


\section{References}

1. Bahn RS (2003) Clinical review 157: Pathophysiology of Graves' ophthalmopathy: the cycle of disease. J Clin Endocrinol Metab 88: 1939-1946.

2. Pedersen I, David M (2008) MicroRNAs in the immune response. Cytokine 43: 391-394.

3. Mitchell PS, Parkin RK, Kroh EM, Fritz BR, Wyman SK, et al. (2008) Circulating microRNAs as stable blood-based markers for cancer detection. Proc Natl Acad Sci U S A 105: 10513-10518.

4. Abou-Zeid A, Saad M, Soliman E (2011) MicroRNA 146a expression in rheumatoid arthritis: association with tumor necrosis factor-alpha and disease activity. Genet Test Mol Biomarkers 15: 807-812.

5. Wang H, Peng W, Ouyang X, Li W, Dai Y (2012) Circulating microRNAs as candidate biomarkers in patients with systemic lupus erythematosus. Transl Res 160: 198-206.

6. Waschbisch A, Atiya M, Linker RA, Potapov S, Schwab S, et al. (2011) Glatiramer acetate treatment normalizes deregulated microRNA expression in relapsing remitting multiple sclerosis. PLoS One 6: e24604.

7. Nahid MA, Pauley KM, Satoh M, Chan EK (2009) miR-146a is critical for endotoxin-induced tolerance: IMPLICATION IN INNATE IMMUNITY. J Biol Chem 284: 34590-34599.

8. Dai R, Phillips RA, Zhang Y, Khan D, Crasta O, et al. (2008) Suppression of LPS-induced Interferon-gamma and nitric oxide in splenic lymphocytes by select estrogen-regulated microRNAs: a novel mechanism of immune modulation. Blood 112: 4591-4597.

9. Taganov KD, Boldin MP, Chang KJ, Baltimore D (2006) NF-kappaB-dependent induction of microRNA miR-146, an inhibitor targeted to signaling proteins of innate immune responses. Proc Natl Acad Sci U S A 103: 12481-12486.

10. Niimoto $T$, Nakasa $T$, Ishikawa $M$, Okuhara A, Izumi B, et al. (2010) MicroRNA-146a expresses in interleukin-17 producing $\mathrm{T}$ cells in rheumatoid arthritis patients. BMC Musculoskelet Disord 11: 209.

11. Kim SE, Yoon JS, Kim KH, Lee SY (2012) Increased serum interleukin-17 in Graves' ophthalmopathy. Graefes Arch Clin Exp Ophthalmol 250: 1521-1526.

12. Han R, Smith TJ (2006) T helper type 1 and type 2 cytokines exert divergent influence on the induction of prostaglandin E2 and hyaluronan synthesis by interleukin-1beta in orbital fibroblasts: implications for the pathogenesis of thyroid-associated ophthalmopathy. Endocrinology 147: 13-19.

13. Miossec P (2009) IL-17 and Th17 cells in human inflammatory diseases. Microbes Infect 11: 625-630.
14. Bettelli E, Korn, T, Oukka M, Kuchroo VK (2008) Induction and effector functions of T(H)17 cells. Nature 453: 1051-1057.

15. Iwakura Y, Nakae S, Saijo S, Ishigame H (2008) The roles of IL-17A in inflammatory immune responses and host defense against pathogens. Immunol Rev 226: 57-79.

16. Wong CK, Lit LC, Tam LS, Li EK, Wong PT, et al. (2008) Hyperproduction of IL-23 and IL-17 in patients with systemic lupus erythematosus: implications for Th17-mediated inflammation in auto-immunity. Clin Immunol 127: 385-393.

17. Hueber AJ, Asquith DL, Miller AM, Reilly J, Kerr S, et al. (2010) Mast cells express IL-17A in rheumatoid arthritis synovium. J Immunol 184: 3336-3340.

18. Matusevicius D, Kivisakk P, He B, Kostulas N, Ozenci $\mathrm{V}$, et al. (1999) Interleukin-17 mRNA expression in blood and CSF mononuclear cells is augmented in multiple sclerosis. Mult Scler 5: 101-104.

19. Bullens DM, Truyen E, Coteur L, Dilissen E, Hellings PW, et al. (2006) IL-17 mRNA in sputum of asthmatic patients: linking $\mathrm{T}$ cell driven inflammation and granulocytic influx? Respir Res 7: 135.

20. Boldin MP, Taganov KD, Rao DS, Yang L, Zhao JL, et al. (2011) miR-146a is a significant brake on autoimmunity, myeloproliferation, and cancer in mice. $J$ Exp Med 208: 1189-1201.

21. Maitra U, Davis S, Reilly CM, Li L (2009) Differential regulation of Foxp3 and IL-17 expression in CD4 T helper cells by IRAK-1. J Immunol 182: 5763-5769.

22. Pauley KM, Satoh M, Chan AL, Bubb MR, Reeves WH, et al. (2008) Upregulated miR-146a expression in peripheral blood mononuclear cells from rheumatoid arthritis patients. Arthritis Res Ther 10: R101.

23. Nakasa T, Miyaki S, Okubo A, Hashimoto M, Nishida $\mathrm{K}$, et al. (2008) Expression of microRNA-146 in rheumatoid arthritis synovial tissue. Arthritis Rheum 58: 1284-1292.

24. Wang G, Tam LS, Li EK, Kwan BC, Chow KM, et al. (2010) Serum and urinary cell-free MiR-146a and MiR155 in patients with systemic lupus erythematosus. $J$ Rheumatol 37: 2516-2522.

25. Bernecker C, Lenz L, Ostapczuk MS, Schinner S, Willenberg H, et al. (2012) MicroRNAs miR-146a1, miR-155 2, and miR-200al are regulated in autoimmune thyroid diseases. Thyroid 22: 1294-1295.

26. Xia P, Fang X, Zhang ZH, Huang Q, Yan KX, et al. (2012) Dysregulation of miRNA146a versus IRAK1 induces IL-17 persistence in the psoriatic skin lesions. Immunol Lett 148: 151-162. 